REVESCO. Revista de Estudios Cooperativos

ISSN: $1885-8031$

https://dx.doi.org/10.5209/REVE.77444

\title{
Estudio de las relaciones de empleo en las cooperativas españolas
}

\author{
Francisco Rincón Roldán ${ }^{1}$
}

Recibido: 29 de enero de 2021 / Aceptado: 28 de junio de 2021 / Publicado: 17 de septiembre de 2021

Resumen. Este trabajo pretende analizar las relaciones de empleo predominantes en las cooperativas españolas. Partimos de la tipología de relaciones de empleo que compara las expectativas que el empleador tiene sobre el empleado y la oferta de incentivos que hace, para analizar el impacto de las mismas en el diseño de las prácticas de gestión de recursos humanos de las cooperativas estudiadas. La información necesaria se obtuvo a través de un cuestionario que respondieron el Director/a de recursos humanos y el Administrador/a de 124 empresas cooperativas. Se aplicaron técnicas estadísticas descriptivas como análisis factorial, estudio de conglomerados y test no paramétrico de Kruskal-Wallis. Los resultados obtenidos sugieren que las relaciones de empleo de Mutual Investment y Overinvestment afectan y condicionan significativamente la gestión de recursos humanos de las cooperativas de la muestra estudiada. En cambio, las relaciones de empleo de Quasi-spot Contract y Underinvestment fomentan en menor medida las prácticas de recursos humanos contenidas en el modelo AMO. De esta manera, se confirma que el tipo de relación de empleo predominante en una cooperativa afecta al diseño de su sistema de recursos humanos.

Palabras clave: Relaciones de Empleo; Gestión de Recursos Humanos; Cooperativas; Economía Social.

Claves Econlit: B55; O15; E24; J29.

\section{[en] Study of employment relationships in spanish cooperatives}

\begin{abstract}
This paper aims to analyze the predominant employment relationships in Spanish cooperatives. We start from the typology of employment relationships that compares the employer's expectations of the employee and the incentives offered, in order to analyze the impact of these expectations on the design of human resource management practices in the cooperatives studied. The necessary information was obtained through a questionnaire answered by the Human Resources Director and the Administrator of 124 cooperative companies. Descriptive statistical techniques such as factor analysis, cluster analysis and the Kruskal-Wallis nonparametric test were applied. The results obtained suggest that Mutual Investment and Overinvestment employment relationships significantly affect and condition the human resources management of the cooperatives in the sample studied. On the other hand, Quasi-spot Contract and Underinvestment employment relationships promote to a lesser extent the human resources practices contained in the AMO model. Thus, it is confirmed that the type of employment relationship predominant in a cooperative affects the design of its human resources system.
\end{abstract}

Keywords: Employment Relationships; Human Resource Management; Cooperatives; Social Economy.

Sumario. 1. Introducción. 2. Marco teórico. 3. Metodología. 4. Resultados. 5. Conclusiones. 6. Referencias bibliográficas.

Cómo citar. Rincon-Roldan, F. (2021) Estudio de las relaciones de empleo en las cooperativas españolas. REVESCO. Revista de Estudios Cooperativos, vol. 139, e77444. https://dx.doi.org/10.5209/reve.77444.

\section{Introducción}

En los últimos años la gestión de los recursos humanos ha ganado importancia en la consecución de una excelencia empresarial, favoreciendo la mejora de las condiciones laborales de los empleados y afectando en su compromiso con la organización, ocupando además una posición central en la mayor parte de las teorías y de los enfoques sobre la dirección de empresas (Céspedes Lorente \& Cano Guillén, 2003). Los múltiples cambios producidos en la estructura del mercado laboral, así como los cambios organizativos internos, como el aplanamiento de las estructuras o la externalización del trabajo, han hecho que las empresas dediquen más esfuerzos a gestionar su recurso humano de manera más eficiente (Rubery, Earnshaw, Marchington, Cooke, \& Vincent, 2002). Cambios que a su vez han afectado a las relaciones que se producen en las organizaciones,

1 Universidad Pablo de Olavide, España.

Dirección de correo electrónico: fjrinrol@upo.es. 
fundamentalmente a la relación comprendida entre empleado y empleador, que denominamos Relaciones de Empleo (Tsui \& Wu, 2005; Wang, Tsui, Zhang, \& Ma, 2003).

La presente investigación considera que el modelo de Relaciones de Empleo (RE) de una organización puede ser útil y añade información al diseño del sistema de recursos humanos de una organización, ya que a través de las RE podemos estudiar como las decisiones organizativas infieren en el comportamiento de sus empleados, a través de las expectativas de rendimiento y de los incentivos que ofrece la empresa (Peel \& Boxall, 2005; Lopez-Cabrales \& Valle Cabrera, 2020). Por tanto, la principal cuestión de investigación de este artículo sería ¿Qué Relaciones de Empleo están más presentes en las cooperativas españolas? Para ello, emplearemos para realizar la presente investigación una muestra de empresas cooperativas, las cuales priorizan el desarrollo económico y social sostenible en su finalidad, por lo que intuimos que las RE que en ellas se generan, tienen una clara orientación hacia dicho desarrollo.

La actual sociedad demanda un cambio de paradigma económico global, donde las empresas tienen un papel fundamental ya que son gestoras y consumidoras de gran cantidad de recursos, se relacionan con otros grupos (gobiernos, accionistas, empleados) y sus acciones deben ir encaminadas hacia un desarrollo sostenible incorporando en su estrategia la sostenibilidad corporativa. Al ser la sostenibilidad el enfoque empresarial que demanda la actual sociedad (Cavagnaro \& Curiel, 2017), el análisis de los principios y valores que la hacen posible se presenta como ineludible (Barea Tejeiro, 2008). Por ello, la elección de este sector se debe además de por su importante crecimiento, por la importancia que tienen los principios y valores en de las entidades cooperativas, así como su clara orientación a generar modelos de gestión sostenibles: social, económico y ambiental (Sunley \& Pinch, 2012). Autores como Cheney, Santa Cruz, Peredo, \& Nazareno (2014) afirman que las empresas que son propiedad de los trabajadores, como es el caso de las cooperativas, tienen un importante papel que desempeñar en la reformulación y reconfiguración de la economía en su conjunto, poniendo sobre la mesa formas alternativas sobre gobernanza y gestión empresarial.

Es necesario resaltar que los principios que rigen a las entidades de economía social, sugieren que las organizaciones deben colocar en el centro de sus políticas a las personas (Fontela, 2017; Marcuello \& Gil, 2008). Ello nos hace plantearnos como la búsqueda de mejores resultados de una organización cooperativa puede ser alcanzada a través de la interiorización de los empleados de valores y principios donde las organizaciones colocan en el centro de sus políticas los interés individuales y sociales por encima de los económicos (Brown, Dillard, \& Marshall, 2006). Los empleados que se consideran reconocidos muestran una mayor implicación con los objetivos de su organización (De Prins, Beirendonck, De Vos, \& Segers, 2014).

Previamente se hace necesario matizar la particularidad de las RE que pueden ofrecer las organizaciones cooperativas. El presente estudio considera que esta RE se produce entre los socios o socios-trabajadores, que sí toman parte en el control y gestión de la cooperativa, con los trabajadores que no ocupan la figura de socio y quienes prestan sus servicios a cambio de una contraprestación (Cuadrado Serrán \& Ciruela Lorenzo, 2015).

Esta cuestión de investigación es relevante, ya que a pesar del interés del desarrollo económico y sostenible en las organizaciones cooperativas, observamos ausencia de estudios sobre la incidencia de las RE en el diseño de sus prácticas de gestión de recursos humanos. En este sentido, Schramm (2011) sostiene que la rentabilidad de una organización está estrechamente relacionada con la forma en la que sus empleados trabajan. Por ello, puede ser relevante estudiar el papel de la relación entre empleado y empleador, que puede incidir en el rendimiento de una empresa y en un mayor desarrollo sostenible.

En coherencia con todo lo anterior, un primer objetivo de este artículo será presentar un modelo teórico que vincule las RE que se producen en una organización cooperativa, y que rigen el funcionamiento de las entidades sociales, con la búsqueda de un modelo empresarial más sólido. De Prins, Beirendonck, De Vos, \& Segers (2014) consideran que la gestión de los recursos humanos está influenciada por las relaciones de trabajo que se producen en una organización, y por tanto estimulan su desarrollo económico y sostenible. Un segundo objetivo del presente capítulo sería analizar cuál de las RE existentes en la muestra estudiada favorece más la implantación de determinadas prácticas de gestión de recursos humanos.

Este artículo se estructura de la siguiente manera. En primer lugar, se plantea un marco teórico a partir de la literatura existente, planteando la relevancia del estudio de la gestión de los recursos humanos y las Relaciones de Empleo. A continuación, se presenta la investigación empírica, a través de la cual se realiza un estudio descriptivo sobre la muestra de cooperativas españolas. Finalmente, se presentan las conclusiones y discusión de los resultados obtenidos. Así como las limitaciones del estudio y sus líneas futuras de investigación. 


\section{Marco Teórico}

\subsection{Prácticas de Gestión de Recursos Humanos: El modelo AMO}

La gestión de los recursos humanos tiene como principal objetivo maximizar el rendimiento del empleado así como su compromiso con la organización, incidiendo consecuentemente en el rendimiento empresarial (Guest, 2002). De entre los numerosos enfoques o perspectivas que giran en torno a la gestión de recursos humanos destaca el Enfoque Universalista, el cual considera que la empresa cuenta con unas determinadas prácticas de alto rendimiento, denominadas "High Performance Work System (HPWS)" en la gestión de los recursos humanos, que siempre inciden positivamente en los resultados de la misma, independientemente de otros elementos internos o externos a la propia organización (Huselid, 1995; Osterman, 1995; Pfeffer, 2005). Este enfoque se apoya en diversas teorías, tales como la Teoría del Capital Humano y la Teoría de los Recursos y las Capacidades (Youndt \& Snell, 2004). De forma, que la primera teoría sostiene que las habilidades, destrezas y conocimientos de los empleados proporcionan a la empresa valor económico, por ello la inversión en capital humano se justifica en el rendimiento que estos empleados aportan a través de su productividad. Por otra parte, la Teoría de los Recursos y las Capacidades, sostiene que las personas juegan un papel clave en el desarrollo y mantenimiento de la ventaja competitiva de la empresa, ya que son consideradas recursos internos y valiosos de la misma (Huselid, 1995).

Sin embargo y tras innumerables investigaciones sobre la relación entre gestión de recursos humanos y rendimiento (Kramar, 2014; Pfeffer, 2005; Siletti, Guerci, Cirella, Shani, \& Radaelli, 2015), aún no existe un consenso claro sobre cuáles son las prácticas de recursos humanos específicas que forman parte de las llamadas $H P W S$, ya que las mismas pueden variar en función de factores como el entorno, el tamaño o la consistencia interna (Ehrnrooth \& Björkman, 2012). Esta falta de consenso ha generado un cierto vacío sobre qué prácticas de recursos humanos funcionan mejor para lograr un mayor rendimiento organizativo, dando lugar a la llamada "Black Box" de la gestión de recursos humanos (Innocenti, Pilati, \& Peluso, 2011; Messersmith, Patel, Lepak, \& Gould-Williams, 2011; Savaneviciene \& Stankeviciute, 2010). Esta "Black Box" ha sido estudiada por numerosos investigadores, quienes han concluido que el modelo AMO (Ability, Motivation and Opportunity) propuesto por Bailey (1993) y posteriormente ampliado (Appelbaum, Bailey, Berg, \& Kalleberg, 2000), puede ser considerado como una herramienta útil para descifrar dicha caja y entender mejor la relación existente entre la gestión de recursos humanos y el rendimiento empresarial (Savaneviciene \& Stankeviciute, 2010; Siletti et al., 2015).

El modelo AMO contempla que no sólo los factores contextuales pueden influir en los resultados positivos de las organizaciones, afirma que las características individuales, las afinidades y circunstancias personales de los empleados inciden en el rendimiento organizativo (Paauwe \& Boselie, 2005). Bailey (1993) sugirió que el rendimiento de un empleado precisa de tres elementos: las habilidades (Ability) necesarias para desarrollar su trabajo, una motivación (Motivation) que aumente su compromiso con la organización y finalmente la organización debe ofrecerles la oportunidad (Opportunity) de participar e involucrarse a través de una mayor autonomía o trabajo en equipo. El actual modelo económico y social requiere que las organizaciones sustenten su ventaja competitiva en su capital humano, aumentando de esta forma su eficiencia, rentabilidad y sostenibilidad (Aykan, 2017; Sharma \& Henriques, 2005).

Desde su aparición el modelo AMO ha sido estudiado y desarrollado ampliamente ofreciendo un amplio estudio sobre la relación entre gestión de recursos humanos y rendimiento empresarial (Appelbaum, Bailey, Berg, \& Kalleberg, 2001; Ehrnrooth \& Björkman, 2012; Paauwe \& Boselie, 2005). El modelo se compone de tres dimensiones básicas, con una fuerte base psicológica (Kroon, Van De Voorde, \& Timmers, 2013), relacionadas con las características individuales de los empleados a través de la mejora de sus conocimientos, comportamientos y capacidades que redunden en mejores resultados organizativos.

En primer lugar la dimensión de Ability, tiene como objetivo mejorar los conocimientos, habilidades y destrezas de los empleados (Fu, Flood, Bosak, Morris, \& O’Regan, 2013). La organización proporciona a sus empleados mejores habilidades para desempeñar su trabajo (Savaneviciene \& Stankeviciute, 2010), a través de prácticas de recursos humanos tales como: el reclutamiento y selección, la formación, entre otras (Katou \& Budhwar, 2010; Kehoe \& Wright, 2013).

La segunda dimensión, la Motivation, está relacionada con la necesidad de realización del trabajador en su empleo a través de una adecuada gestión de la motivación tanto extrínseca como intrínseca, donde ambas promueven el compromiso del empleado con su organización (Munteanu, 2014). La organización puede fomentar la motivación de sus empleados a través de prácticas de recursos humanos tales como: el sistema de compensación, un adecuado diseño de la carrera profesional, entre otras (Choi \& Yoon, 2015; Katou, 2008; Kehoe \& Wright, 2013).

El modelo AMO introduce una tercera dimensión, la Opportunity, centrada fundamentalmente en la participación del empleado en la organización, a través de un entorno laboral que promueve la autonomía, el trabajo en equipo y la comunicación abierta y horizontal. Ofreciendo la oportunidad a los empleados de aportar ideas, sugerencias, involucrarse, en definitivita generar un valor añadido (Block \& Pickl, 2014). La 
organización puede ofrecer oportunidades a sus empleados a través de prácticas de recursos humanos tales como: el trabajo en equipo, diseñando puestos más autónomos, mejorando las condicionales laborales y personales a través de prácticas como los procesos de participación o la evaluación del desempeño, entre otras (Bainbridge, 2015; Block \& Pickl, 2014; Paauwe \& Boselie, 2005).

Finalmente autores como Farndale, Hope-Hailey, \& Kelliher (2011) argumentan que el modelo AMO fomenta el compromiso y el rendimiento de los empleados, debido a que estos interpretan como la empresa invierte en sus empleados y aumentando la sensación de apoyo percibido. Añaden además que este modelo tiene un mayor impacto cuando sus tres dimensiones se encuentran alineadas y funcionan como un sistema integrado. Por lo que podemos concluir que el modelo AMO es considerado el corazón de la gestión estratégica de los recursos humanos (Katou, 2008). La organización fomenta de esta forma el compromiso de sus empleados guiando sus actitudes y comportamientos hacía un mayor rendimiento y en consecuencia los empleados sienten la necesidad de recompensar a su organización por el apoyo y respeto recibido, a través de sus habilidades, motivaciones y oportunidades ofrecidas (Katou \& Budhwar, 2010).

\subsection{Las Relaciones de Empleo}

Como hemos visto anteriormente, la rentabilidad de una empresa está asociada a la forma en que se gestionan los empleados de la misma, ya que estos pueden comprometerse en el desarrollo económico, social y/o ambiental de su organización (Ehnert, 2009; Lopez-Cabrales \& Valle-Cabrera, 2020). En este sentido, varios estudios han demostrado cómo determinadas prácticas de gestión de recursos humanos contribuyen significativamente a mejorar el comportamiento de los empleados, tales como el trabajo en equipo, acciones formativas específicas, un adecuado sistema de compensación, entre otras (Buller \& McEvoy, 2016; De Prins et al., 2014). Pero esta investigación pretende poner el foco de atención no sólo en las prácticas aisladas de recursos humanos sino en las RE, ya que éstas analizan la relación de intercambio que se produce entre los comportamientos deseados por la dirección de la empresa y los incentivos ofrecidos a cambio. De esta forma, podemos definir RE como aquella relación de intercambio en la que el empleador contrata fuerza de trabajo con la finalidad de producir bienes o servicios, para obtener un beneficio propio, donde el empleado presta sus servicios a cambio de una contraprestación, la cual puede o no ser solamente económica (Shore \& Coyle-Shapiro, 2003).

El modelo de Relaciones de Empleo usado en esta investigación es el formulado por los autores Tsui \& Wu (2005), quienes presentan cuatro posibles relaciones de empleo en una organización, desde el punto de vista del empleador, comparando los incentivos que ofrece la empresa frente a las contribuciones esperadas que el manager tiene sobre sus empleados (Figura 1).

Figura. 1. Modelo de Relaciones de Empleo.

\begin{tabular}{|c|c|c|c|}
\hline \multirow{3}{*}{ 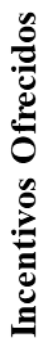 } & \multirow{2}{*}{$\begin{array}{l}\text { Alto } \\
\text { Bajo }\end{array}$} & Overinvestment & Mutual investment \\
\hline & & Quasi-spot contract & Underinvestment \\
\hline & & Bajo & Alto \\
\hline & & Contri & speradas \\
\hline
\end{tabular}

Fuente: Adaptado de Tsui \& Wu (2005)

Tsui \& Wu (2005) destacan la existencia de dos bloques contrapuestos, por un lado, las RE equilibradas y por otro las desequilibradas. En la relación equilibrada se da un ajuste entre el nivel de expectativas que la empresa tiene de las contribuciones que debe hacer el empleado y la oferta de incentivos que realiza la empresa a cambio. Encontramos dos tipos de RE en equilibrio. La primera denominada "Mutual Investment" se caracteriza porque el empleador espera de sus empleados altos niveles de compromiso y rendimiento, así como un bajo nivel de absentismo. En contraprestación, les ofrece un gran número de incentivos como formación y desarrollo profesional, participación en la toma de decisiones, un atractivo paquete retributivo y una estabilidad en el empleo en el largo plazo. La segunda RE en equilibrio sería la denominada como "Quasi-spot Contract", donde el empleador espera del empleado un conjunto reducido de contribuciones, centrados en la ejecución de la tarea sin necesidad de fomentar el compromiso, esta relación tiene una clara 
orientación al corto plazo, con sistemas retributivos poco interesantes para el empleado, poca seguridad y escasos beneficios sociales. Se trata de una relación equilibrada debido a que el bajo nivel de incentivos que se ofrece a los empleados es coherente o justo en relación al bajo nivel de contribuciones o rendimiento que se espera de ellos.

Podemos añadir que, a través de la RE de Mutual Investment la organización invierte en aquellos trabajadores que mayores contribuciones pueden generar y que además juegan un papel relevante en la rentabilidad de la organización. Consideran que los empleados están potencialmente preparados para implementar y desarrollar dentro de la organización comportamientos orientados a maximizar el rendimiento organizativo (Stone, 2000). A su vez, los empleados responden ante las altas contribuciones de su organización con altos niveles de rendimiento, así como un elevado compromiso con los objetivos de su organización. En cambio, la RE de Quasi-spot Contract, tiene una clara orientación al corto plazo, empleando la mano de obra necesaria en función de sus necesidades productivas. Esta RE se produce con facilidad cuando el mercado laboral está descompensado y es fácil obtener empleados a cambio de escasos incentivos. En esta RE el recurso humano no es considerado como una ventaja competitiva, más bien se presenta como un recurso para lograr un objetivo de producción. La organización tiene bajas expectativas sobre el rendimiento del empleado y por ello les ofrece escasos incentivos (Tsui, Pearce, \& Porter, 1997), se considera por tanto que los empleados ubicados en esta relación de empleo ejercen una escasa aportación al rendimiento de una organización.

El segundo bloque de RE planteado por Tsui \& Wu (2005) es aquel donde no existe equilibrio entre la oferta de incentivos y las expectativas de rendimiento que tiene el empleador. La primera RE de este tipo sería la denominada como "Overinvestment" producida cuando la organización ofrece a sus empleados incentivos superiores a las contribuciones que estos realizan, básicamente debido a que las expectativas de la empresa son mayores que el rendimiento real actual de sus empleados, ya que se confía en que estos contribuirán en mayor medida en el futuro. La segunda alternativa sería la denominada como "Underinvestment" donde el empleado recibe a cambio de un alto rendimiento una escasa contraprestación por parte de la organización, situación que se produce con frecuencia en momentos de crisis económica y financiera, donde la organización encuentra una clara justificación para exigir a sus empleados el máximo y ofrecer a cambio la mínima contraprestación.

Respecto a las relaciones de Overinvestment, autores como Shaw, Dineen, Fang, \& Vellella (2009) encontraron evidencias del importante impacto de esta RE sobre el comportamiento y compromiso de los empleados. Consideran que a través de altas contribuciones los empleados pueden desarrollar su potencial y convertirse en una clara ventaja competitiva de la organización que promueva mayor rendimiento y desarrollo económico. Esta RE se aplicaría con personas con un alto potencial y contribuciones inicialmente bajas, debido a que están empezando a adquirir y desarrollar los conocimientos y habilidades básicas necesarias para alcanzar un mayor rendimiento de su organización. Este tipo de relación se basa en la confianza de la empresa en las contribuciones futuras y esperadas del empleado, donde el capital humano agregado juega un importante papel (Bornay-Barrachina, de la Rosa-Navarro, Cabrales, \& Valle, 2012). Finalmente, la RE de Underinvestment se caracteriza por la escasa inversión que realiza la empresa en su personal, exigiendo en cambio un alto compromiso y rendimiento. Esta situación de desequilibrio es muy común con altos índices de desempleo o en situaciones de crisis económica, donde la empresa encuentra fácilmente mano de obra en el mercado. Sin embargo, este intercambio no puede perdurar en el largo plazo ya que provocaría en la organización altas tasas de abandono y mal clima laboral. Así pues, los incentivos ofrecidos por la organización en materia de formación, retribución o seguridad laboral no van más allá del mínimo legal exigido. La búsqueda de un modelo organizativo rentable no tiene cabida en organizaciones que se rigen por principios y valores donde no se invierte en su recurso humano (Tsui \& Wu, 2005), consideramos por tanto que una organización debe invertir en su recurso humano para desarrollar capacidades que potencien su desarrollo económico y social sostenible en el tiempo.

Podemos plantearnos en qué medida el tipo de RE condiciona el grado de rentabilidad empresarial, ya que las RE son claves para promover el desarrollo de las organizaciones (Boxall, 2014; Lopez-Cabrales \& ValleCabrera, 2020; Romanelli, 2013). Asumimos que las empresas cuyo objetivo es lograr una mayor rentabilidad esperarán un mayor compromiso de sus empleados, quienes aporten sus conocimientos, actitudes y habilidades, para lograr una organización más rentable (Renwick, Redman, \& Maguire, 2012). Si el objetivo de las organizaciones es ser más rentable económica, social y ambientalmente, deben fomentar un mayor compromiso de sus empleados, para beneficiarse de sus conocimientos y habilidades, generando una actitud proactiva que fomente el desarrollo de la organización. Basándonos en la teoría de la reciprocidad de Gouldner (1960), las organizaciones deben invertir en sus empleados para fomentar las actitudes y comportamientos deseados, ya que los empleados se sentirán obligados a devolver esta inversión recibida en el desarrollo de su trabajo, conocimientos y habilidades. 


\section{Metodología}

\subsection{Muestra y procedimiento}

La unidad de análisis empleada para esta investigación son las empresas de Economía Social, dentro de las cuales estudiamos las cooperativas, por ser las entidades más representativas, atendiendo a su mayor presencia, generación de empleo y facturación. Según el informe publicado por Cooperatives Europe titulado "The power of Cooperation - Cooperatives Europe Key Figures 2015", el movimiento cooperativo en Europa suma más de 140 millones de ciudadanos que forman parte de alguna cooperativa Europea, con cerca de 180 mil empresas cooperativas que generan más de 4,7 millones de empleos (Quintana, 2016).

En España las entidades de Economía Social tienen como objetivo principal el servicio a las personas (Monzón Campos, 2006) y están presentes en gran parte de los sectores de actividad, con una presencia que supera las 42.100 entidades y que generan un empleo directo e indirecto de más de 2,1 millones de personas y una facturación superior a los 150.000 millones de euros, datos que equivalen al 10\% del PIB español (CEPES, 2019). Según los datos estadísticos ofrecidos por la Secretaría de Estado de Empleo y Economía Social del Gobierno de España en 2019 existían más de 20.800 organizaciones cooperativas que emplean a más de 300.000 trabajadores en España. Destacando significativamente su capacidad para crear y mantener empleo, el cual se ha visto incrementado en el último trimestre del año 2019 en más de un $10 \%$.

La muestra seleccionada fueron 598 cooperativas extraídas del informe "Empresas relevantes de la Economía Social 2017-2018" publicado a principios de 2019 por la Confederación Empresarial Española de Economía Social (CEPES), con una plantilla mínima de 50 empleados. Se establece este requisito de dimensión (mínimo 50 empleados), ya que la literatura en materia de recursos humanos establece la dificultad de las organizaciones pequeñas para tener implantadas políticas formalizadas de gestión de recursos humanos (Huselid, 1995; Pfeffer, 1998).

La muestra empleada representa una parte importante de la población objeto de estudio. Según se desprenden de los datos de la Dirección General del Trabajo Autónomo de la Economía Social y de la Responsabilidad Social de las Empresas perteneciente al Ministerio de Trabajo y Economía Social del gobierno de España, en el último trimestre de 2018 (excluido el régimen de autónomo) existían un total de 12.078 cooperativas inscritas a la Seguridad Social, generando más de 240.000 puestos de trabajo y con presencia en todos los sectores de producción de nuestro sistema económico productivo. Además, se hace preciso resaltar como el movimiento cooperativo está presente en la amplía geografía del territorio español, modelo que asocia a personas que tienen necesidades o intereses comunes y para cuya satisfacción desarrollan actividades empresariales, caracterizadas por la imputación de los resultados económicos a los socios que las conforman.

Con la finalidad de analizar que la muestra de la presente investigación es representativa de la población de las cooperativas existentes en el territorio español, se ha realizado un contraste de igualdad de distribuciones entre ambos grupos. Para ello realizamos un contraste sobre la bondad de ajuste a través de la prueba de Kolmogorov-Smirnov. Mediante el contraste de bondad de ajuste de Kolmogorv-Smirnov se prueba si los datos de una muestra proceden, o no, de una determinada distribución de probabilidad. Para ello se comparó la función de distribución acumulada calculada a partir de los datos de la muestra, con la función de distribución acumulada teórica de la distribución con la que se compara, en nuestro caso la población (Massey, 1951). Para verificar que la muestra sigue una distribución similar a la de la población se aplicó un contraste de hipótesis no paramétrico. Específicamente, en la presente investigación realizamos la prueba de bondad de ajuste de Kolmogorov-Smirnov, a través del análisis de las diferencias entre las probabilidades acumuladas de la población y la muestra, agrupando las cooperativas por tipo (Tabla 1), ya que esta variable se encuentra tanto en los datos de la Dirección General del Trabajo Autónomo de la Economía Social y de la Responsabilidad Social de las Empresas perteneciente al Ministerio de Trabajo y Economía Social del gobierno de España, como en el informe elaborado por CEPES. De este modo:

\section{$H_{0}$ : La distribución de probabilidad de la población y de la muestra son homogéneas}

\section{$H_{1}$ : La distribución de probabilidad de la población y de la muestra no son homogéneas}

$$
\begin{gathered}
D=\max \left|F_{N}\left(x_{i}\right)-F_{n}\left(x_{i}\right)\right|=0,053 \\
D_{(0.05 ; 598)}=\frac{1,36}{\sqrt{n}}=0,056
\end{gathered}
$$


Dado que el estadístico $(0,053)$ es menor que el valor tabulado $(0,056)$, no se rechaza la hipótesis de comportamiento homogéneo de las distribuciones de probabilidad de la muestra y la población, para un nivel de significación del $5 \%$ y una muestra de 598 cooperativas. Por tanto, podemos concluir que la muestra empleada en la presente investigación sigue una distribución similar a la de la población.

Tabla. 1. Tipo de Cooperativas de la población y la muestra.

\begin{tabular}{l|c|c}
\hline \multicolumn{1}{c|}{ DISTRIBUCIÓN POR TIPO } & POBLACIÓN & MUESTRA \\
\hline Trabajo asociado & 6.805 & 330 \\
\hline Agrarias & 3.190 & 133 \\
\hline Explotación comunitaria de la tierra & 479 & 31 \\
\hline Servicios y comercio & 377 & 39 \\
\hline Consumidores y usuarios & 276 & 20 \\
\hline Enseñanza & 291 & 10 \\
\hline Transportistas & 219 & 7 \\
\hline Viviendas & 91 & 3 \\
\hline Crédito & 57 & 7 \\
\hline Mar & 16 & 2 \\
\hline Sanitarias & 2 & 11 \\
\hline Otros & 275 & $\mathbf{5 9 8}$ \\
\hline TOTAL & $\mathbf{1 2 . 0 7 8}$ & \\
\hline
\end{tabular}

Fuente: Elaboración propia

En referencia al contexto de investigación, según los datos ofrecidos por la Dirección General del Trabajo Autónomo, de la Economía Social y de la Responsabilidad Social de las Empresas dependiente del Ministerio de Trabajo y Economía Social, en el año 2018 el movimiento cooperativo experimentó un importante auge y consolidación con una inscripción de 1.540 nuevas sociedades cooperativas y más de 8.334 socios. Ello demuestra la importancia del sector elegido para desarrollar la presente investigación.

Se obtuvieron respuestas de 124 cooperativas que supone una tasa de respuesta en referencia a la muestra del $20,7 \%$. Estos datos suponen además una tasa de respuesta de casi el $13 \%$ sobre la población de 958 empresas de Economía Social que conforman el informe de CEPES. Este dato se considerada aceptable debido al bajo índice de respuesta que generalmente se obtiene con la técnica de recogida de datos mediante la técnica del cuestionario (Tomaskovic-Devey, Leiter, \& Thompson, 1994).

La recogida de información para desarrollar el estudio se realizó a través de un cuestionario online a partir de la revisión de la literatura, que permitió garantizar la confidencialidad de las repuestas y agilizar el proceso de recogida de datos (Merino, Pintado, Sánchez, \& Grande, 2010). Es necesario destacar que el envío de los cuestionarios fue reforzado con un seguimiento telefónico a las empresas cooperativas que han participado en el estudio, a través del cual se agilizó y dinamizó la participación de los miembros de las cooperativas seleccionadas. Para el diseño y gestión del cuestionario se utilizó el software "LimeSurvey". Se trata de una aplicación de software libre para el diseño y envío de encuestas online. La herramienta posee múltiples funciones para el diseño de cuestionarios abiertos o cerrados sin necesidad de un amplio conocimiento informático. Además, permite controlar el acceso y posterior seguimiento de la encuesta a través de invitaciones y recordatorios de acceso a la encuesta mediante un link enviado a través de un mail de presentación o recordatorio.

El cuestionario fue estructurado en 3 secciones que corresponden con las 2 variables principales de esta investigación (Prácticas de Gestión de Recursos Humanos contenidas en el Modelo AMO y Relaciones de Empleo) y una tercera que hace referencia a las variables sociodemográficas. En total, el cuestionario completo empleado constaba de 55 ítems, las escalas empleadas se describen a continuación.

\subsection{Medición de las variables}

Todas las variables contenidas en la presente investigación provienen de escalas previamente válidas por la literatura de referencia, asegurándonos de esta forma la validez de la información recabada. Todas las escalas puntúan del 1 al 5, siendo 1 "Totalmente en desacuerdo" y 5 "Totalmente de acuerdo".

En relación con la medición del modelo $\mathrm{AMO}$, se ha empleado y adaptado la escala de medida ampliamente usada y contrastada, propuesta por Gardner, Moynihan, \& Wright (2001), que incluye tres bloques de prácticas de gestión de recursos humanos que mejoran tanto las habilidades como la motivación y ofrece oportunidades a los empleados, con un total de 14 ítems. Como ejemplo de algunos ítems empleados 
destacamos los siguientes: para medir las prácticas de habilidad: "Los candidatos se someten a entrevistas estructuradas antes de ser contratados" o "Los resultados de la evaluación del desempeño se utilizan para determinar las acciones formativas futuras"; para medir las prácticas de motivación: "La retribución de los empleados se basa en el rendimiento de su trabajo" o "Los empleados reciben comisiones individuales en función de su rendimiento individual" y las prácticas de oportunidad: "Los empleados son involucrados en procesos de participación como: grupos de mejora de la calidad, grupos de resolución de problemas, grupos de sugerencias, etc." о "Los empleados reciben periódicamente comunicación formal sobre su desempeño en la organización".

Respecto a la medición de las RE, se ha empleado y adaptado la escala propuesta por Wang et al., (2003), quienes proponen dos dimensiones: las contribuciones esperadas de los empleados por el empleador y los incentivos ofrecidos por los empleadores, recogidos en 29 ítems. Como ejemplo de algunos ítems empleados, para las contribuciones esperadas destacamos: "La empresa espera de sus empleados la mejora continua de los métodos y procedimientos en el trabajo" o "La empresa espera de sus empleados que ayuden a resolver los problemas asociados al trabajo". Respecto a los ítems empleados para medir los incentivos ofrecidos por la organización destacamos: "La empresa ofrece el desarrollo de un plan de carrera para sus empleados" o "La empresa ofrece niveles salariales competitivos".

Para probar la fiabilidad y validez de las medidas, se realizó primero un Análisis Factorial Exploratorio (AFE), con el objetivo de estudiar la dimensionalidad de las variables, aplicando para ello el método de rotación Varimax (Luque Martínez, 2000). Todas las medidas usadas en la investigación mostraron una aceptable validez interna discriminante y convergente. Posteriormente se realizó un Análisis Factorial Confirmatorio (AFC), y se estudiaron las cargas factoriales. La tabla 2 muestra cada ítem con su carga factorial, $R^{2}$, así como los datos principales obtenidos del AFC y la información correspondiente a la bondad del ajuste, los cuales están dentro de los límites establecidos (Hu \& Bentler, 1999). Algunas cargas factoriales ofrecen valores por debajo de 0.7 (Hair, Black, Babin, \& Anderson (2010), pero se consideraron aceptables, ya que su valor es cercano a los estándares establecidos y aportan mayor ajuste al modelo. La fiabilidad y consistencia interna han sido medidas a través del Alfa de Cronbach (Tabla 3), con valores muy aceptables comprendidos entre 0.7 y 0.9 (Hair et al., 2010). Finalmente, se muestra cómo la Varianza Media Extraída (AVE) es en la mayoría de los casos mayor que 0.5 , o con valores muy cercanos, confirmando por tanto la validez convergente (Hair et al., 2010).

Tabla. 2. Ítems, cargas factoriales, $R^{2}$, y resultados del Análisis Factorial Confirmatorio.

\begin{tabular}{|c|c|c|c|}
\hline Items & $\begin{array}{c}\text { Cargas } \\
\text { Factoriales }\end{array}$ & $R^{2}$ & $\begin{array}{l}\text { Resultados } \\
\text { AFC }\end{array}$ \\
\hline \multicolumn{4}{|l|}{ RELACIONES DE EMPLEO $^{1}$} \\
\hline \multicolumn{4}{|l|}{ Ítems para medir las contribuciones esperadas por el empleador: } \\
\hline \multicolumn{4}{|l|}{ Factor uno: Professional managerial duties } \\
\hline $\begin{array}{l}\text { 1. Mejorar continuamente los procedimiento y métodos del } \\
\text { trabajo }\end{array}$ & .573 & .33 & $\chi^{2}=125.2448$ \\
\hline 2. Adoptar nuevas ideas y métodos de trabajo & .627 & .39 & $p=.00000$ \\
\hline 3. Intentar ayudar para que sus subordinados resuelvan & .739 & & RMSEA = \\
\hline problemas relacionados con el trabajo & & .55 & 1036 \\
\hline 4. La adopción de nuevas ideas y métodos en el trabajo & .783 & .61 & $\mathrm{CFI}=.8489$ \\
\hline 5. Que ayuden a resolver los problemas asociados al trabajo & .712 & .51 & $\mathrm{GFI}=.8617$ \\
\hline $\begin{array}{l}\text { 6. Que se preocupen por la mejora y desarrollo de sus } \\
\text { habilidades y destrezas }\end{array}$ & .795 & .63 & \\
\hline $\begin{array}{l}\text { 7. Que desarrollen comportamientos de cooperación con otros } \\
\text { compañeros de su departamento }\end{array}$ & .771 & .59 & \\
\hline $\begin{array}{l}\text { 8. Una buena comunicación con sus supervisores y } \\
\text { subordinados }\end{array}$ & .570 & .32 & \\
\hline 9. La toma de iniciativa para llevar a cabo nuevas tareas & $\begin{array}{l}\text { Descartado } \\
\text { por el } A F C\end{array}$ & -- & \\
\hline
\end{tabular}

\section{Factor dos: Personal work ethics}

1. Que mejoren continuamente sus conocimientos y habilidades de gestión

3. Que contribuyan de manera desinteresada al desarrollo futuro de la empresa 
5. Llevar a cabo tareas extras no contempladas en su actividad cotidiana

6. Asumir y llevar a cabo las decisiones tomadas por la empresa
Descartado

por el AFC

Descartado

por el AFC

Ítems para medir las contribuciones ofrecidas por el empleador:

Factor uno: Developmental rewards

1. Un trato justo a cada empleado

2. Oportunidades para que los trabajadores pongan de manifiesto su potencial

3. La consideración seria de sus sugerencias y comentarios

4. El desarrollo de un Plan de Carrera a cada empleado

5. Delegación de responsabilidades dentro de su ámbito de actuación

6. Participación en el proceso de toma de decisiones de la empresa

7. Valorar sus opiniones en las decisiones que afecten a toda la empresa

8. Procurar su satisfacción en el trabajo

9. Respeto a la dignidad humana

10. Formación necesaria para futuros trabajos y el desarrollo de su carrera

.544

.707

.758

.697

.610

.743

.660

.682

Descartado

por el $A F C$

.598
$.30 \quad \chi^{2}=185.9748$

.50

$p=1.354 \mathrm{e}-13$

RMSEA =

$\begin{array}{lc}.57 & .1036 \\ .49 & \mathrm{CFI}=.7934\end{array}$

.37

$\mathrm{GFI}=.8171$

\section{Factor dos: Materialistic rewards}

1. Bonos y comisiones competitivos

.590

.35

2. Niveles salariales competitivos

.700

3. Beneficios sociales relacionados con la salud (Seguro médico, Seguro Dental, etc.)

.905

4. Beneficios sociales relacionados con la vivienda (Ayudas al Alquiler, Seguro de Vivienda, etc.)

$.794 \quad .63$

\section{MODELO AMO ${ }^{2}$}

\section{Habilidad}

1. Los candidatos se someten a entrevistas estructuradas antes de ser contratados

.756

2. Los candidatos son sometidos a pruebas escritas antes de ser contratados

.904

3. Los resultados de la evaluación del desempeño se utilizan para determinar las acciones formativas futuras

4. Los empleados reciben ayudas para financiar sus estudios

\section{Motivación}

1. La retribución de los empleados se basa en el rendimiento de su trabajo

2. Los empleados reciben comisiones individuales en función de su rendimiento individual

.599

3. Los empleados reciben comisiones grupales, en función del rendimiento del grupo

.691

.46

.816

.771

4. Los empleados reciben comisiones en función de la mejora de los resultados de la empresa

5. Los empleados son evaluados formalmente al menos una vez al año

6. Los empleados cualificados tienen la oportunidad de ascender a puestos de mayor responsabilidad y remuneración dentro de la empresa

\section{Oportunidad}

1. Los empleados tienen un sistema de quejas y sugerencias justo y razonable

2. Los empleados son involucrados en procesos de participación 
como: grupos de mejora de la calidad, grupos de resolución de problemas, grupos de sugerencias, etc.

3. Los empleados se comunican con compañeros de otros departamentos para resolver problemas y cumplir objetivos 4. Los empleados reciben periódicamente comunicación formal sobre su desempeño en la organización

.698

.744

Fuente: Elaboración propia

Escalas: ${ }^{1}$ (Wang et al., 2003), ${ }^{2}$ (Gardner et al., 2001).

Tabla. 3. Alfa de Cronbach, AVE y Correlaciones.

\begin{tabular}{|c|c|c|c|c|c|}
\hline $\begin{array}{l}\text { Alfa de Cronbach } \\
\text { AVE }\end{array}$ & $\begin{array}{l}.855 \\
.511 \\
\end{array}$ & $\begin{array}{l}.724 \\
.528 \\
\end{array}$ & $\begin{array}{l}.853 \\
.488 \\
\end{array}$ & $\begin{array}{l}.768 \\
.630 \\
\end{array}$ & $\begin{array}{l}.911 \\
.874 \\
\end{array}$ \\
\hline \multirow[b]{2}{*}{ 1. Professional managerial duties } & 1 & 2 & 3 & 4 & 5 \\
\hline & 1 & & & & \\
\hline 2. Personal work ethics & 122 & 1 & & & \\
\hline 3. Developmental rewards & $.620^{* * *}$ & $.197^{*}$ & 1 & & \\
\hline 4. Materialistic rewards & $.103^{*}$ & $.076^{*}$ & $.268^{* * *}$ & 1 & \\
\hline 5. Modelo AMO & $.511^{* *}$ & $.493^{*}$ & $.631^{* *}$ & $.772^{* *}$ & 1 \\
\hline
\end{tabular}

Fuente: Elaboración propia

** Correlation is significant at the 0.01 level (2-tailed).

*Correlation is significant at the 0.05 level (2-tailed).

\subsection{Análisis de datos}

Con el objetivo de reducir el sesgo de respuesta asociado con el uso de un solo encuestado (Gerhart, Wright, McMahan, \& Snell, 2000), el cuestionario fue remitido a dos miembros de la cooperativa, concretamente a la persona con responsabilidades en la dirección: Gerente, o Administradora/a y al Director/a de recursos humanos, en caso de no existir esta figura en la cooperativa se remitió a la persona con responsabilidades en dicha área.

\section{Sesgo de no respuesta}

Para medir el "sesgo de no respuesta" en la muestra obtenida, se realizó un análisis ANOVA que examina la información obtenida a través de los cuestionarios en dos momentos diferentes del trabajo de campo, entre las cooperativas que respondieron de forma temprana y aquellas que lo hicieron más tarde, en las últimas semanas del mismo. Por tanto la muestra quedo dividida en dos grupos: Primero.- cooperativas que habían contestado antes de diciembre de 2019: 71 puestos con responsabilidades en dirección y 67 puestos con funciones en recursos humanos. Y aquellas cooperativas que contestaron a partir del 1 de diciembre: 53 puestos con responsabilidades en dirección y 57 puestos con funciones en recursos humanos. Dividiendo por tanto la muestra en dos ondas, donde la las respuestas de la segunda onda proyecta la tendencia de los encuestados que no han participado en el estudio (Filion, 1976). De esta forma se consideró que las cooperativas que responden de forma tardía están más próximas a las cooperativas que no han reportado ninguna información, que a las que responden de forma temprana. La tabla 4 muestra que efectivamente no existen diferencias significativas con respecto a las variables planteadas en el presente estudio. Concluyendo por tanto que el "sesgo de no respuesta" no es un problema para los datos de la investigación.

Tabla. 4. Análisis ANOVA - Sesgo de no respuesta.

\begin{tabular}{ccc}
\hline Constructo & ANOVA $(\mathbf{F})$ & Significancia \\
\hline Professional managerial duties & 1.040 & $0.426^{\mathrm{ns}}$ \\
Personal work ethics & 0.716 & $0.721^{\mathrm{ns}}$ \\
Developmental rewards & 0.441 & $0.983^{\text {ns }}$ \\
Materialistic rewards & 1.154 & $0.315^{\mathrm{ns}}$ \\
AMO Model & 1.113 & $0.527^{\mathrm{ns}}$ \\
\hline
\end{tabular}

Fuente: Elaboración propia

Nota: $\mathrm{ns}=$ no significativo. 


\section{Agregación de datos}

Tal y como hemos señalado anteriormente, para llevar a cabo la presente investigación se seleccionaron dos informantes de cada organización, a la persona con responsabilidades en materia de dirección: Presidente/a, Gerente, Director/a General y en segundo lugar al Director/a de recursos humanos o persona que aun no ocupando ese puesto desempeña las funciones adscritas al mismo. Obtuvimos ambas respuestas de 124 cooperativas (248 respuestas), por lo que se hace necesario estudiar la agregación de los datos de la muestra. Se analizó el acuerdo intra-grupo a través del ratio interagreement $\left(r_{w g}\right)$ (James, Demaree, \& Wolf, 1984), a través del cual podemos medir el nivel de acuerdo entre los dos informantes de cada organización y constatar por tanto la emergencia del constructo a un nivel superior. Seguidamente se estudió la fiabilidad de la agregación empleando los coeficientes interclase (ICC1 e ICC2) propuestos por Bliese (2000), valorando por tanto la consistencia relativa de una respuesta entre los distintos encuestados (Tabla 5).

Tabla. 5. Ratio Interagreement y Ratios ICC.

\begin{tabular}{c|c|c|c}
\hline Variable & $\mathbf{r}_{\text {wg }}{ }^{*}$ & ICC1 $^{* *}$ & ICC2 $^{* * * *}$ \\
\hline Relaciones de Empleo & 0,842112535 & 0,082 & 0,708 \\
Modelo AMO & 0,792265671 & 0,092 & 0,751 \\
\hline
\end{tabular}

Fuente: Elaboración propia

Notas: ${ }^{*} r_{\mathrm{wg}}>0,7$ (James et al., 1984); ${ }^{* *} \mathrm{ICC} 1>0,05 ;{ }^{* * *} \mathrm{ICC} 2>0,7$ (Bliese, 2000).

\section{Resultados}

Para clasificar las empresas según el tipo de relación de empleo predominante, se realizó un análisis exploratorio de los datos. El gráfico de dispersión (Gráfico 2) creado a partir de las RE estudiadas, permitió identificar claramente cuatro grupos, a partir del comportamiento de los valores medios de los datos obtenidos, resultado soportado por el modelo planteado por Tsui \& Wu (2005). Posteriormente se asignó cada empresa a uno de los cuatro tipos de RE en función de las puntuaciones obtenidas en cada uno de los cuatro factores que configuran las RE: professional managerial duties; personal work ethics; developmental rewards y materialistic rewards.

Gráfico. 2. Gráfico de dispersión.

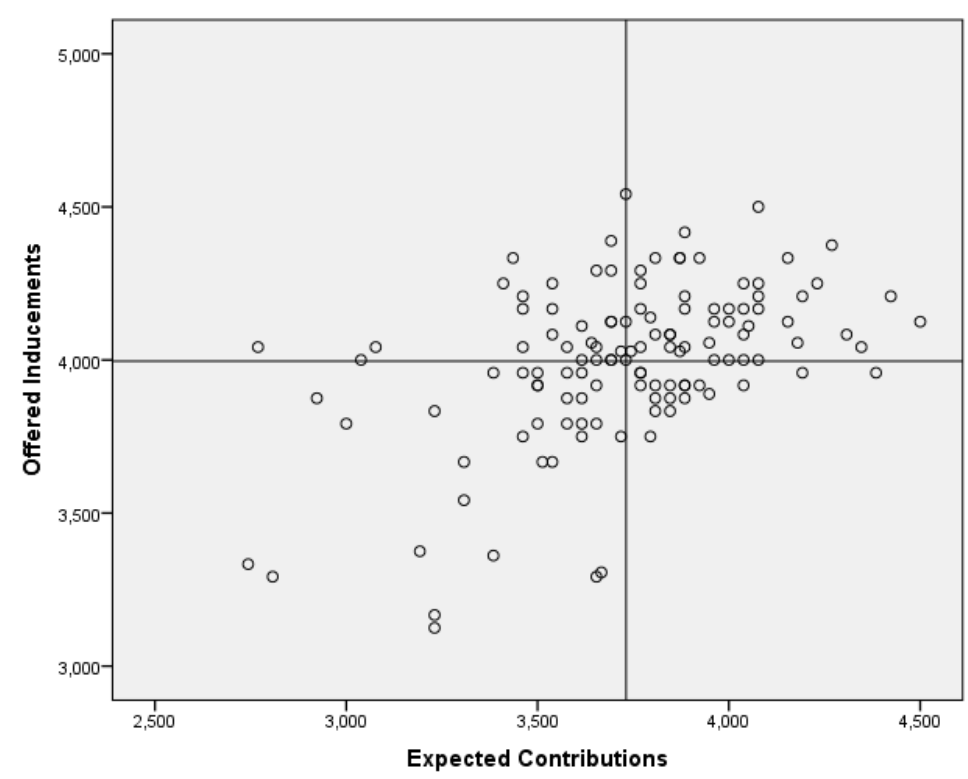

Fuente: IBM SPSS Statistics 27.0

La tabla 6 muestra las puntuaciones medias de las dos dimensiones que conforman el modelo de RE en cada uno de los cuatro conglomerados, así como la puntuación media de la muestra que fue tomada como punto de referencia. Un mayor grupo de empresas (41 cooperativas) superó al resto en las dimensiones de contribuciones esperadas e incentivos ofrecidos. Concretamente la puntuación obtenida por este grupo en los 
cuatro factores fue la siguiente: profesional managerial duties $(4.205>4.007)$, personal work ethic (4.041>3.948), developmental rewards $(4.240>4.000)$ y finalmente en materialistic rewards $(3.445>3.151)$. Debido a que tanto las expectativas como los incentivos superan la media del resto de dimensiones, se considera a este grupo dentro de la RE de "Mutual Investment" (MI). El segundo grupo con mayor número de empresas agrupadas (38 cooperativas) ofrecía las puntuaciones más bajas de la muestra en las dimensiones de expectativas e incentivos al se denominó como "Quasi-spot Contract" (QSC), con las siguientes puntuaciones: $(3.713<4.007 ; 3.779<3.948 ; 3.689<4.000 ; 2.844<3.151)$. El tercer grupo (23 cooperativas) ofrecía altas puntuaciones en contribuciones esperadas $(4.156>4.007 ; 4.134>3.948)$ y bajas puntuaciones en incentivos ofrecidos $(3.935<4.000 ; 2.866<3.151)$, debido a sus características, considerando a este grupo como "Underinvestment" (UI). Finalmente, el último grupo (22 cooperativas) ofrecía puntuaciones bajas en contribuciones esperadas $(3.992<4.007 ; 3.875<3.948)$ y altas puntuaciones en incentivos ofrecidos $(4.159>4.000 ; 3.409>3.151)$, este último grupo fue denominado "Overinvestment" (OI). Los valores de los cuatro conglomerados muestran diferencias significativas según los resultados de la ANOVA ( $F$ Value), empleado para medir las diferencias entre los cuatro grupos que aparecen en la muestra.

Tabla. 6. Dimensiones de RREE: Análisis Clúster.

\begin{tabular}{|c|c|c|c|c|c|c|}
\hline & Variable & $\begin{array}{c}\text { Muestra } \\
\text { Promedio }\end{array}$ & Grupo & Mean & SD & $\begin{array}{c}F \\
\text { Value }\end{array}$ \\
\hline \multirow{8}{*}{$\begin{array}{l}\text { CONTRIBUCIONES } \\
\text { ESPERADAS }\end{array}$} & \multirow{2}{*}{$\begin{array}{l}\text { Professional } \\
\text { Managerial Duties }\end{array}$} & 4.007 & Mutual Investment & 4.205 & .267 & \multirow{4}{*}{$\begin{array}{r}60.272 \\
(.000)\end{array}$} \\
\hline & & \multirow{7}{*}{3.948} & $\begin{array}{l}\text { Quasi-Spot } \\
\text { Contract }\end{array}$ & 3.713 & .399 & \\
\hline & \multirow{6}{*}{$\begin{array}{l}\text { Personal } \\
\text { Ethics }\end{array}$} & & Underinvestment & 4.156 & .234 & \\
\hline & & & Overinvestment & 3.992 & .083 & \\
\hline & & & Mutual Investment & 4.041 & .332 & \multirow{4}{*}{$\begin{array}{r}64.407 \\
(.000)\end{array}$} \\
\hline & & & $\begin{array}{l}\text { Quasi-Spot } \\
\text { Contract }\end{array}$ & 3.779 & .382 & \\
\hline & & & Underinvestment & 4.134 & .368 & \\
\hline & & & Overinvestment & 3.875 & .308 & \\
\hline \multirow{8}{*}{$\begin{array}{l}\text { INCENTIVOS } \\
\text { OFRECIDOS }\end{array}$} & \multirow{4}{*}{$\begin{array}{l}\text { Developmental } \\
\text { rewards }\end{array}$} & 4.000 & Mutual Investment & 4.240 & .250 & \multirow{4}{*}{$\begin{array}{l}69.096 \\
(.000)\end{array}$} \\
\hline & & & $\begin{array}{l}\text { Quasi-Spot } \\
\text { Contract }\end{array}$ & 3.689 & .327 & \\
\hline & & & Underinvestment & 3.935 & .294 & \\
\hline & & & Overinvestment & 4.159 & .233 & \\
\hline & \multirow{4}{*}{$\begin{array}{l}\text { Materialistic } \\
\text { rewards }\end{array}$} & 3.151 & Mutual Investment & 3.455 & .568 & \multirow{4}{*}{$\begin{array}{l}75.942 \\
(.000)\end{array}$} \\
\hline & & & $\begin{array}{l}\text { Quasi-Spot } \\
\text { Contract }\end{array}$ & 2.844 & .590 & \\
\hline & & & Underinvestment & 2.866 & .563 & \\
\hline & & & Overinvestment & 3.409 & .492 & \\
\hline
\end{tabular}

Fuente: Elaboración propia

De cara a complementar el análisis realizado y dado que la muestra se dividió en 4 clusters de reducido tamaño muestral que impedían la realización de pruebas de inferencia estadística en cada grupo, se realizó un análisis descriptivo complementario para ver cómo se comportan las prácticas AMO dentro de cada cluster, realizando una aproximación descriptiva sobre las variables incluidas en los modelos y a las características de la muestra (Hair, 2011). La tabla 7 recoge para cada cluster de relación de empleo los valores medios, moda y deviación típica de las variables estudiadas en este artículo: prácticas del modelo AMO: las que favorecen el desarrollo de habilidades, motivación y oportunidad. Estos parámetros permiten caracterizar la muestra y aportar una visión general del comportamiento de los datos en relación a la centralidad y la dispersión de cada submuestra formada por las diferentes relaciones de empleo.

De los datos contenidos en la tabla 7 podemos destacar como los valores medios de las prácticas AMO son más elevados en las relaciones de empleo de Mutual Investment y Overinvestment, ello demuestra que las organizaciones que establecen relaciones de empleo a través de las cuales ofrecen interesantes incentivos a sus empleados, consiguen modular de forma positiva sus prácticas de gestión de recursos humanos. Además, podemos observar cómo los valores modales de las variables Habilidad, Motivación y Oportunidad de las relaciones de empleo Quasi-spot Contract y Underinvestment están generalmente un punto por debajo de los valores de las relaciones de empleo Mutual Investment y Overinvestment, lo que nos lleva a concluir 
que estás prácticas de recursos humanos están más presentes en las relaciones de empleo que ofrecen incentivos atractivos a los empleados y por tanto fomentan las habilidades, generan motivación y promueven las oportunidades de sus empleados.

Tabla. 7. Estadísticos descriptivos.

\begin{tabular}{lclccccc}
\hline \multicolumn{1}{c}{ Cluster } & $\boldsymbol{N}$ & Variable & Media & Moda & $\begin{array}{c}\text { Desv. } \\
\text { Típica }\end{array}$ & Min. & Max. \\
\hline \multirow{2}{*}{ MUTUAL INVESTMENT } & \multirow{2}{*}{41} & Habilidad & 3,95 & 4 & 0,62 & 1 & 5 \\
& & Motivación & 3,79 & 4 & 0,60 & 1 & 5 \\
& & Oportunidad & 4,07 & 4 & 0,47 & 1 & 5 \\
\hline \multirow{2}{*}{ QUASI-SPOT } & \multirow{2}{*}{ CONTRACT } & Habilidad & 2,77 & 3 & 0,61 & 1 & 5 \\
& \multirow{2}{*}{ OVERINVESTMENT } & Motivación & 2,67 & 3 & 0,57 & 1 & 5 \\
& & Oportunidad & 2,86 & 3 & 0,49 & 1 & 5 \\
\hline \multirow{2}{*}{ UNDERINVESTMENT } & \multirow{2}{*}{22} & Habilidad & 3,68 & 4 & 0,65 & 1 & 5 \\
& & Motivación & 4,48 & 3 & 0,65 & 1 & 5 \\
& & Oportunidad & 3,79 & 3 & 0,56 & 1 & 5 \\
\hline \multirow{2}{*}{23} & Habilidad & 2,89 & 3 & 0,36 & 1 & 5 \\
& & Motivación & 2,71 & 2 & 0,47 & 1 & 5 \\
& & Oportunidad & 2,63 & 2 & 0,67 & 1 & 5 \\
\hline
\end{tabular}

Fuente: Elaboración propia

Además, con la finalidad de analizar si las diferencias entre clusters encontradas en la tabla 7 son significativas se realizó un test no paramétrico de Kruskal-Wallis (Tabla 8). Esta técnica es utilizada para estudiar si las variables empleadas en la población de estudio tienen un comportamiento similar en cuanto a sus funciones de distribución. Como también puede verse en la tabla 8 , la columna de estadísticos de prueba obtenidos con un nivel de significancia del 0,05 , podemos concluir que existen evidencias significativas de la existencia de diferencias entre las medias de los diferentes clusters estudiados. Además, podemos observar cómo, atendiendo al rango promedio, los cluster formados por las relaciones de empleo Mutual Investment y Overinvestment alcanzan los valores medios más elevados de las variables estudiadas: habilidad, motivación y oportunidad. En cambio, los clusters de Quasi-spot Contract y Underinvestment, obtienen valores medios inferiores. Siendo el último cluster citado, el Underinvestment, el que ofrece valores más bajos, lo que nos lleva a concluir que aquellas organizaciones que ofrecen incentivos muy por debajo de las expectativas de sus empleados, cuentan con una escasa relevancia de las prácticas contenidas en el modelo AMO.

Tabla. 8. Test de Kruskal-Wallis.

\begin{tabular}{|c|c|c|c|c|}
\hline Variable & RREE & $n$ & Rango promedio & Estadísticos de prueba ${ }^{a, b}$ \\
\hline & 1 & 41 & 79,09 & Chi-cuadrado $=39,426$ \\
\hline Prácticas: & 2 & 22 & 69,92 & $g l=3$ \\
\hline \multirow[t]{3}{*}{ Habilidad } & 3 & 38 & 60,77 & Sig. asintótica $=, 000$ \\
\hline & 4 & 23 & 22,33 & \\
\hline & Total & 124 & & \\
\hline & 1 & 41 & 72,59 & Chi-cuadrado $=8,123$ \\
\hline Prácticas: & 2 & 22 & 65,17 & $g l=3$ \\
\hline \multirow[t]{3}{*}{ Motivación } & 3 & 38 & 52,73 & Sig. asintótica $=, 044$ \\
\hline & 4 & 23 & 49,46 & \\
\hline & Total & 124 & & \\
\hline & 1 & 41 & 81,07 & Chi-cuadrado $=55,112$ \\
\hline Prácticas: & 2 & 22 & 71,03 & $g l=3$ \\
\hline \multirow[t]{3}{*}{ Oportunidad } & 3 & 38 & 63,70 & Sig. asintótica $=, 000$ \\
\hline & 4 & 23 & 14,15 & \\
\hline & Total & 124 & & \\
\hline
\end{tabular}

Fuente: Elaboración propia

Notas: RREE: Relación de Empleo; (1)Mutual Investment; (2)Overinvestment; (3)Quasi-spot Contract (4)Underinvestment. a. Prueba de Kruskal-Wallis; b. Variable de agrupación: RREE. 


\section{Conclusiones}

Los resultados de esta investigación permiten una mejor comprensión de los efectos subyacentes entre las RE de una organización cooperativa y sus prácticas de gestión de recursos humanos. En primer lugar, se demuestra empíricamente la influencia significativa de las RE Mutual Investment y Overinvestment sobre las prácticas de gestión de recursos humanos contenidas en el Modelo AMO. Este resultado es coherente con los principios que rigen a las entidades cooperativas, que sugieren que las organizaciones deben colocar en el centro de sus políticas a los empleados (Morrison, 2002). Los empleados que se consideran reconocidos muestran una mayor implicación con los objetivos de su organización, como puede ser la búsqueda de una mayor rentabilidad (De Prins et al., 2014). Las entidades de Economía Social cuentan con una serie de principios y valores éticos que componen su cultura empresarial y que condiciona su estrategia, que se concreta en una RE con altos incentivos. Debido a su enfoque transformador, estas organizaciones usan y promueven nuevos patrones de gestión, producción, distribución de ingresos y excedentes, así como un nuevo marco de relaciones sociales y laborales (Coraggio, 2009). Se postulan además como un importante instrumento estratégico, no sólo por sus cualidades económicas y de gestión, sino también por su compromiso con la sociedad (Borzaga, Salvatori, \& Bodini, 2017; Tomás Carpi, 1997).

Además, los datos obtenidos de la aproximación descriptiva sobre las variables estudiadas muestran disparidad en referencia al papel de las prácticas AMO en las diferentes relaciones de empleo. Destacan como las relaciones de Mutual Investment y Overinvestment ofrecen los valores modales más elevados. Lo que nos lleva a concluir que las relaciones de empleo que ofrecen interesantes incentivos consiguen influir de forma positiva en el diseño de sus prácticas de recursos humanos. Sin embargo, las relaciones de empleo de Quasi-spot Contract y Underinvestment ofrecen valores modales más bajos en las tres dimensiones del modelo AMO, lo que nos lleva a concluir que estás prácticas de recursos humanos carecen de relevancia en las relaciones de empleo con escasos incentivos hacías los empleados.

Los resultados del análisis no paramétrico realizado, a través del test de Kruskal-Wallis, permiten determinar que las variables empleadas en la población de estudio tenían un comportamiento similar en cuanto a sus funciones de distribución. De los resultados obtenidos se desprenden las siguientes conclusiones: primero los clusters de Mutual Investment y Overinvestment alcanzan los valores medios más elevados en las tres dimensiones del modelo AMO, lo que indica que estos tipos de relaciones de empleo ofrecen el mejor marco para que las cooperativas formulen y diseñen su estrategia de recursos humanos. En cambio, los clusters formados por las relaciones de Quasi-spot Contract y Underinvestment ofrecen los valores medios más bajos, constatando que aquellas cooperativas que ofrecen escasos incentivos a sus empleados difícilmente pueden emplear las prácticas de gestión de recursos humanos para alcanzar una mayor rentabilidad.

Como mayores contribuciones de este artículo podemos destacar en primer lugar, que la orientación de determinadas RE en las cooperativas ejerce un impacto positivo en su gestión de recursos humanos, por lo que consideramos de sumo interés seguir profundizando en este estudio. Además, la proliferación de investigaciones sobre recursos humanos y rentabilidad está originando una diversidad de enfoques de la misma, produciéndose una importante fragmentación del concepto y sus dimensiones (Schuler, Rasche, Etzion, \& Newton, 2017). En segundo lugar, destacamos la relevancia del sector donde se ha llevado a cabo la investigación, el sector de empresas cooperativas, que cuenta con un importante crecimiento en el panorama económico y social europeo.

No obstante, esta investigación no está exenta de limitaciones, cuya superación a su vez configuran líneas futuras de investigación. Si bien el sector cooperativo es interesante, sus relaciones de empleo podrían ser diferentes a las predominantes en otros sectores industriales o de servicios, por lo que se puede plantear como una línea futura de investigación extrapolar el estudio realizado a otros sectores. Otra limitación del trabajo y propuesta de estudio sería el contexto geográfico, la investigación se ha circunscrito a España, puede ser interesante ampliar el contexto geográfico a otros países y estudiar cómo pueden afectar los aspectos interculturales a la gestión de los recursos humanos y a las RE de una organización. Finalmente, una limitación referida al cuestionario empleado es la relacionada con el uso de la escala de medida de 1-5 puntos. El uso de esta escala trae consigo la obtención de una reducida variabilidad de los datos obtenidos, que consecuentemente dificultan la interpretación de los mismos.

Es necesario destacar como las RE más presentes en la muestra estudiada son las de Mutual Investment (41 cooperativas) y Quasi-spot Contract (38 cooperativas), las cuales forman el grupo de definidas por los autores Tsui \& Wu (2005) como Relaciones de Empleo Equilibradas, que recordemos son aquellas donde se da un ajuste entre el nivel de expectativas que la empresa tiene de las contribuciones que debe hacer el empleado y la oferta de incentivos que realiza la empresa a cambio. Este resultado es coherente con la idiosincrasia de las cooperativas españolas, ya que en ellas podemos encontrar la figura del socio trabajador, donde los empleados reciben interesantes incentivos a cambio de su alta implicación en la gestión de la cooperativa. Pero también podemos encontrar una relación puramente contractual, donde el empleado de la cooperativa que no figura como socio, realiza una mera transacción de su rendimiento a través de una 
contraprestación. Las organizaciones cooperativas muestran ambas RE y ambas se muestran válidas y eficientes, ya que están contempladas en los principios, características y valores de las entidades del sector en las que se encuadran.

En conclusión, este trabajo demuestra que las RE contempladas en la presente investigación, Mutual Investment y Overinvestment afectan significativamente a la configuración de la gestión de los recursos humanos de las empresas cooperativas. Ello puede llevar a confirmar la importancia que confieren las entidades del sector social a su recurso humano, considerado como una importante ventaja competitiva sostenible (Truong \& Barraket, 2018). Ahora son las organizaciones las que deben aprovechar estas sinergias para desarrollar comportamientos más rentables y sostenibles.

\section{Referencias bibliográficas}

Appelbaum, E., Bailey, T., Berg, P., \& Kalleberg, A. (2000) Manufacturing Advantage: Why High-Performance Work Systems Pay Off. The Academy of Management Review (Vol. 26). https://doi.org/10.2307/259189.

Appelbaum, E., Bailey, T., Berg, P., \& Kalleberg, A. L. (2001) Do high performance work systems pay off? Research in the Sociology of Work, 10, pp. 85-107. https://doi.org/10.1016/S0277-2833(01)80022-4.

Aykan, E. (2017) Gaining a Competitive Advantage through Green Human Resource Management. In Corporate Governance and Strategic Decision Making. https://doi.org/10.5772/intechopen.69703.

Bailey, T. R. (1993) Discretionary Effort and the Organization of Work: Employee Participation and Work Reform Since Hawthorne. Teachers College and Conservation of Human Resources, Columbia University.

Bainbridge, H. (2015) Devolving people management to the line: How different rationales for devolution influence people management effectiveness. Personnel Review, 44(6), pp. 847-865. https://doi.org/10.1108/PR-10-2013-0193.

Barea Tejeiro, J. (2008) El concepto científico de economía social desde la perspectiva actual. Temas Para El Debate, 167, pp. 23-26. Retrieved from https://dialnet.unirioja.es/servlet/articulo?codigo=2966756.

Bliese, P. D. (2000) Within-group agreement, non-independence, and reliability: Implications for data aggregation and analysis. In Multilevel theory, research, and methods in organizations: Foundations, extensions, and new directions. (pp. 349-381). San Francisco, CA, US: Jossey-Bass.

Block, J., \& Pickl, S. (2014) The Mystery of Job Performance: A System Dynamics Model of Human Behavior The Black-Box of HRM. The 32nd International Conference of the System Dynamics Society, (July), pp. 1-28. Retrieved from

http://scholar.google.com/scholar?hl=en\&btnG=Search\&q=intitle:Strategic+Human+Resource+Management+:+A+ Balanced+Approach\#0.

Bornay-Barrachina, M., de la Rosa-Navarro, D., Cabrales, A., \& Valle, R. (2012) Employment Relationships and Firm Innovation: The Double Role of Human Capital. British Journal of Management, 23. https://doi.org/10.1111/j.14678551.2010.00735.x.

Borzaga, C., Salvatori, G., \& Bodini, R. (2017) International Labour Organization. International Organization. https://doi.org/10.1017/S0020818300022694.

Boxall, P. (2014) The future of employment relations from the perspective of human resource management. Journal of Industrial Relations, 56, pp. 578-593. https://doi.org/10.1177/0022185614527980.

Brown, D., Dillard, J., \& Marshall, R. (2006) Triple Bottom Line: A business metaphor for a social construct. Understanding the Social Dimension of Sustainability. https://doi.org/10.4324/9780203892978.

Buller, P. F., \& McEvoy, G. M. (2016) A Model for Implementing a Sustainability Strategy through HRM Practices. Business and Society Review, 121(4), pp. 465-495. https://doi.org/10.1111/basr.12099.

Cavagnaro, E., \& Curiel, G. (2017) The Three Levels of Sustainability. The Three Levels of Sustainability. Greenleaf Pub. https://doi.org/10.4324/9781351277969.

CEPES. (2019) Análisis del impacto socioeconómico de los valores y principios de la economía social en españa. Gobierno de España, Ministerio de Trabajo, Migraciones y Seguridad Social.

CEPES - Confederación Empresarial Española de la Economía Social. (2019) Empresas más relevantes de la Economía Social 2017-2018.

Céspedes Lorente, J., \& Cano Guillén, C. (2003) Estrategia de negocio y prácticas de recursos humanos en las cooperativas. CIRIEC-España. Revista de Economía Pública, Social y Cooperativa, No 46, pp. 63-84. Retrieved from https://dialnet.unirioja.es/servlet/articulo?codigo=769630.

Cheney, G., Santa Cruz, I., Peredo, A. M., \& Nazareno, E. (2014) Worker cooperatives as an organizational alternative: Challenges, achievements and promise in business governance and ownership. Organization, 21(5), pp. 591-603. https://doi.org/10.1177/1350508414539784.

Choi, M., \& Yoon, H. J. (2015) Training investment and organizational outcomes: a moderated mediation model of employee outcomes and strategic orientation of the HR function. International Journal of Human Resource Management, 26(20), pp. 2632-2651. https://doi.org/10.1080/09585192.2014.1003084.

Coraggio, J. L. (2009) Íconos. Revista de Ciencias Sociales. Íconos: Revista de Ciencias Sociales, ISSN-e 1390-1249, No. 33, 2009, págs. 29-38. FLACSO - Ecuador (Facultad Latinoamericana de Ciencias Sociales). Retrieved from https://dialnet.unirioja.es/servlet/articulo?codigo=3318455. 
Cuadrado Serrán, M., \& Ciruela Lorenzo, A. (2015) Las sociedades cooperativas y laborales como artífices del emprendimiento empresarial. Análisis del perfil del emprendedor de ambas figuras en el contexto de Andalucía. CIRIEC-España. Revista de Economía Pública, Social y Cooperativa, $\mathrm{N}^{\mathrm{o}} \quad 84, \quad$ pp. 5-34. https://doi.org/10.7203/CIRIEC-E.84.13397.

De Prins, P., Beirendonck, L. Van, De Vos, A., \& Segers, J. (2014) Sustainable HRM: Bridging theory and practice through the 'Respect Openness Continuity (ROC)'-model. Management Revue, 25(4), pp. $263-284$. https://doi.org/10.1688/mrev-2014-04-Prins.

Ehnert, I. (2009) Sustainable Human Resource Management. Heidelberg: Physica-Verlag HD. https://doi.org/10.1007/978-3-7908-2188-8.

Ehrnrooth, M., \& Björkman, I. (2012) An Integrative HRM Process Theorization: Beyond Signalling Effects and Mutual Gains. Journal of Management Studies, 49(6), pp. 1109-1135. https://doi.org/10.1111/j.14676486.2012.01055.x.

Farndale, E., Hope-Hailey, V., \& Kelliher, C. (2011) High commitment performance management: The roles of justice and trust. Personnel Review, 40(1), pp. 5-23. https://doi.org/10.1108/00483481111095492.

Filion, F. L. (1976) Exploring and Correcting for Nonresponse Bias Using Follow-ups of Non Respondents. Pacific Sociological Review, 19(3), pp. 401-408. https://doi.org/10.2307/1388756.

Fontela, J. (2017) Las relaciones entre los valores y principios cooperativos y los principios de la normativa cooperativa. REVESCO. Revista de Estudios Cooperativos, Segundo Cuatrimestre, N ${ }^{\mathrm{o}} 124$, pp. $114-127$. https://doi.org/10.5209/REVE.54923.

Fu, N., Flood, P. C., Bosak, J., Morris, T., \& O'Regan, P. (2013) Exploring the performance effect of HPWS on professional service supply chain management. Supply Chain Management. https://doi.org/10.1108/SCM-04-2012$\underline{0118}$.

Gardner, T. M., Moynihan, L. M., \& Wright, P. M. (2001) Beginning to Unlock the Black Box in the HR Firm Performance Relationship: The Impact of HR Practices on Employee Attitudes and Employee Outcomes. Working Paper Series.

Gerhart, B. (2005) Human Resources and Business Performance: Findings, Unanswered Questions, and an Alternative Approach. Management Review, 16(2), pp. 174-185. https://doi.org/10.5771/0935-9915-2005-2-174.

Gerhart, B., Wright, P. M., McMahan, G. C., \& Snell, S. A. (2000) Measurement error in research on human resources and firm performance: How much error is there and how does it influence effect size estimates? Personnel Psychology, 53(4), pp. 803-834. https://doi.org/10.1111/j.1744-6570.2000.tb02418.x.

Gouldner, A. W. (1960) The Norm of Reciprocity: A Preliminary Statement. American Sociological Review, 25(2), pp. 161-178. https://doi.org/10.2307/2092623.

Guest, D. (2002) Human Resource Management, Corporate Performance and Employee Wellbeing: Building the Worker into HRM. Journal of Industrial Relations, 44(3), pp. 335-358. https://doi.org/10.1111/1472-9296.00053.

Hair, J., Black, W., Babin, B., \& Anderson, R. (2010) Multivariate Data Analysis: A Global Perspective. In Multivariate Data Analysis: A Global Perspective (Vol. 7th).

Hair, J. F. (2011) Multivariate Data Analysis: An Overview. In International Encyclopedia of Statistical Science (pp. 904-907). Springer Berlin Heidelberg. https://doi.org/10.1007/978-3-642-04898-2_395.

Hu, L., \& Bentler, P. M. (1999) Cutoff criteria for fit indexes in covariance structure analysis: Conventional criteria versus new alternatives. Structural Equation Modeling, 6(1), pp. 1-55. https://doi.org/10.1080/10705519909540118.

Huselid, M. A. (1995) The Impact Of Human Resource Management Practices On Turnover, Productivity, And Corporate Financial Performance. Academy of Management Journal, 38(3), pp. $635-672$. https://doi.org/10.5465/256741.

Innocenti, L., Pilati, M., \& Peluso, A. M. (2011) Trust as moderator in the relationship between HRM practices and employee attitudes. Human Resource Management Journal, 21(3), pp. 303-317. https://doi.org/10.1111/j.17488583.2010.00151.x.

J. Schramm. (2011) Promoting Sustainability. HR Magazine. Retrieved from https://www.shrm.org/hr-today/news/hrmagazine/Pages/0311focus.aspx.

James, L., Demaree, R., \& Wolf, G. (1984) Estimating Within-Group Interrater Reliability With and Without Response Bias. Journal of Applied Psychology, 69, pp. 85-98. https://doi.org/10.1037/0021-9010.69.1.85.

Katou, A. A. (2008) Measuring the impact of HRM on organisational performance. Journal of Industrial Engineering and Management, 1(2), pp. 119-142. https://doi.org/10.3926/jiem.2008.v1n2.p119-142.

Katou, A. A., \& Budhwar, P. S. (2010) Causal relationship between HRM policies and organisational performance: Evidence from the Greek manufacturing sector. European Management Journal, 28(1), pp. 25-39. https://doi.org/10.1016/j.emj.2009.06.001.

Kehoe, R. R., \& Wright, P. M. (2013) The Impact of High-Performance Human Resource Practices on Employees' Attitudes and Behaviors. Journal of Management, 39(2), pp. 366-391. https://doi.org/10.1177/0149206310365901.

Kramar, R. (2014) Beyond strategic human resource management: is sustainable human resource management the next approach? The International Journal of Human Resource Management, 25(8), pp. $1069-1089$. https://doi.org/10.1080/09585192.2013.816863.

Kroon, B., Van De Voorde, K., \& Timmers, J. (2013) High performance work practices in small firms: A resourcepoverty and strategic decision-making perspective. Small Business Economics, 41(1), pp. 71-91. https://doi.org/10.1007/s11187-012-9425-0. 
Lopez-Cabrales, A., \& Valle-Cabrera, R. (2020) Sustainable HRM strategies and employment relationships as drivers of the triple bottom line. Human Resource Management Review, 30(3), 100689. https://doi.org/https://doi.org/10.1016/j.hrmr.2019.100689.

Luque Martínez, T. (2000) Técnicas De Análisis De Datos En Investigación De Mercados.

Marcuello, C., \& Gil, I. (2008) Los principios cooperativos facilitadores de la innovación: un modelo teórico. REVESCO. Revista de Estudios Cooperativos, No 94, pp. 59-79.

Massey, F. J. (1951) The Kolmogorov-Smirnov Test for Goodness of Fit. Journal of the American Statistical Association, 46(253), pp. 68-78. https://doi.org/10.1080/01621459.1951.10500769.

Merino, M. J., Pintado, T., Sánchez, J., \& Grande, I. (2010) Introducción a La Investigación De Mercados. Retrieved December 25, 2019, from https://www.amazon.es/Introducción-Investigación-Mercados-Librosprofesionales/dp/8415986777.

Messersmith, J. G., Patel, P. C., Lepak, D. P., \& Gould-Williams, J. (2011) Unlocking the black box: Exploring the link between high-performance work systems and performance. Journal of Applied Psychology, 96(6), pp. 1105-1118. https://doi.org/10.1037/a0024710.

Monzón Campos, J. (2006) Economía Social y conceptos afines: fronteras borrosas y ambigüedades conceptuales del Tercer Sector. CIRIEC-España. Revista de Economía Pública, Social y Cooperativa, No 56, pp. 9-24.

Morrison, E. W. (2002) Newcomers' relationships: The role of social network ties during socialization. Academy of Management Journal, 45(6), pp. 1149-1160. https://doi.org/10.2307/3069430.

Munteanu, A.-I. (2014) WHAT MEANS HIGH PERFORMANCE WORK PRACTICES FOR HUMAN RESOURCES IN AN ORGANIZATION? Annals of the University of Petrosani : Economics, 14(1), pp. 243-250.

Osterman, P. (1995) Skill, Training, and Work Organization in American Establishments. Industrial Relations: A Journal of Economy and Society, 34(2), pp. 125-146. https://doi.org/10.1111/j.1468-232X.1995.tb00365.x.

Paauwe, J., \& Boselie, P. (2005) HRM and performance: What next? Human Resource Management Journal. https://doi.org/10.1111/j.1748-8583.2005.tb00296.x.

Peel, S., \& Boxall, P. (2005) When is Contracting Preferable to Employment? An Exploration of Management "and" Worker Perspectives. Journal of Management Studies, 42, pp. 1675-1697. https://doi.org/10.1111/j.14676486.2005.00562.x.

Pfeffer, J. (1998) Seven Practices of Successful Organizations. California Management Review, 40. https://doi.org/10.2307/41165935.

Pfeffer, J. (2005) Changing mental models: HR's most important task. Human Resource Management, 44(2), pp. 123128. https://doi.org/10.1002/hrm.20053.

Quintana, C. (2016) The power of cooperation: Cooperatives Europe Key Figures 2015. Cooperatvies Europe, p. 128. Retrieved from https://coopseurope.coop/resources/projects/power-cooperation-cooperatives-europe-key-figures$\underline{2015}$.

Renwick, D., Redman, T., \& Maguire, S. (2012) Green Human Resource Management: A Review and Research Agenda. International Journal of Management Reviews, 15. https://doi.org/10.1111/j.1468-2370.2011.00328.x.

Romanelli, M. (2013) Organizations and People for Sustainability. Management Dynamics in the Knowledge Economy, 6(1), pp. 117-128. https://doi.org/10.25019/mdke/6.1.07.

Rubery, J., Earnshaw, J., Marchington, M., Cooke, F., \& Vincent, S. (2002) Changing Organisational Forms of the Employment Relationship. Journal of Management Studies, 39, pp. 645-672. https://doi.org/10.1111/1467$\underline{6486.00306}$

Savaneviciene, A., \& Stankeviciute, Z. (2010) The models exploring the "black box" between HRM and organizational performance. Engineering Economics, 21(4), pp. 426-434. https://doi.org/10.5755/j01.ee.21.4.11719.

Schuler, D., Rasche, A., Etzion, D., \& Newton, L. (2017) Corporate Sustainability Management and Environmental Ethics. SSRN Electronic Journal. https://doi.org/10.2139/ssrn.2951788.

Sharma, S., \& Henriques, I. (2005) Stakeholder influences on sustainability practices in the Canadian forest products industry. Strategic Management Journal, 26(2), pp. 159-180. https://doi.org/10.1002/smj.439.

Shaw, J., Dineen, B., Fang, R., \& Vellella, R. (2009) Employee-Organization Exchange Relationships, HRM Practices, and Quit Rates of Good and Poor Performers. Academy of Management Journal, 52, pp. 1016-1033. https://doi.org/10.5465/AMJ.2009.44635525.

Shore, L. M., \& Coyle-Shapiro, J. A.-M. (2003) Editorial: New developments in the employee-organization relationship. Journal of Organizational Behavior, 24(SPEC. ISS.), pp. 443-450. https://doi.org/10.1002/job.212.

Siletti, E., Guerci, M., Cirella, S., Shani, A., \& Radaelli, G. (2015) The Impact of Human Resource Management Practices and Corporate Sustainability on Organizational Ethical Climates: An Employee Perspective. Journal of Business Ethics, 126, pp. 325-342. https://doi.org/10.1007/s10551-013-1946-1.

Stone, L. (2000) When case studies are not enough: The influence of corporate culture and employee attitudes on the success of cleaner production initiatives. Journal of Cleaner Production, 8, pp. 353-359. https://doi.org/10.1016/S0959-6526(00)00037-8.

Sunley, P., \& Pinch, S. (2012) Financing social enterprise: social bricolage or evolutionary entrepreneurialism? Social Enterprise Journal, 8(2), pp. 108-122. https://doi.org/10.1108/17508611211252837.

Tomás Carpi, J. A. (1997) The prospects for the social economy in a changing world. Annals of Public and Cooperative Economics, 68(2), pp. 247-279. https://doi.org/https://doi.org/10.1111/1467-8292.00045. 
Tomaskovic-Devey, D., Leiter, J., \& Thompson, S. (1994) Organizational Survey Nonresponse. Administrative Science Quarterly, 39(3), 439. https://doi.org/10.2307/2393298.

Truong, A., \& Barraket, J. (2018) Engaging workers in resource-poor environments: the case of social enterprise in Vietnam. The International Journal of Human Resource Management, 29(20), pp. 2949-2970. https://doi.org/10.1080/09585192.2018.1479875.

Tsui, A., Pearce, J., \& Porter, L. (1997) Alternative approaches to the employee-organization relationship: Academy of Management Journal.

Retrieved from http://scholar.google.com/scholar?hl=en\&btnG=Search\&q=intitle:Alternative+approaches+to+the+employeeorganization+relationship\#2.

Tsui, A. S., \& Wu, J. B. (2005) The new employment relationship versus the mutual investment approach: Implications for human resource management. Human Resource Management, 44(2), pp. $115-121$. https://doi.org/10.1002/hrm.20052.

Wang, D., Tsui, A. S., Zhang, Y., \& Ma, L. (2003) Employment relationships and firm performance: Evidence from an emerging economy. Journal of Organizational Behavior, 24(SPEC. ISS.), pp. 511-535. https://doi.org/10.1002/job.213.

Youndt, M. A., \& Snell, S. A. (2004) Human Resource Configurations, Intellectual Capital, and Organizational Performance. Journal of Managerial Issues. Pittsburg State University. https://doi.org/10.2307/40604485. 